\title{
Drift compression and final focus for intense heavy ion beams with nonperiodic, time-dependent lattice
}

\author{
Hong Qin and Ronald C. Davidson \\ Plasma Physics Laboratory, Princeton University, Princeton, New Jersey 08543, USA \\ John J. Barnard \\ Lawrence Livermore National Laboratory, Livermore, California 94550, USA
}

Edward P. Lee

Lawrence Berkeley National Laboratory, Berkeley, California 94720, USA

(Received 22 December 2003; published 12 October 2004)

\begin{abstract}
In the currently envisioned configurations for heavy ion fusion, it is necessary to longitudinally compress the beam bunches by a large factor after the acceleration phase. Because the space-charge force increases as the beam is compressed, the beam size in the transverse direction will increase in a periodic quadrupole lattice. If an active control of the beam size is desired, a larger focusing force is needed to confine the beam in the transverse direction, and a nonperiodic quadrupole lattice along the beam path is necessary. In this paper, we describe the design of such a focusing lattice using the transverse envelope equations. A drift compression and final focus lattice should focus the entire beam pulse onto the same focal spot on the target. This is difficult with a fixed lattice, because different slices of the beam may have different perveance and emittance. Four time-dependent magnets are introduced in the upstream of drift compression to focus the entire pulse onto the same focal spot. Drift compression and final focusing schemes are developed for a typical heavy ion fusion driver and for the integrated beam experiment being designed by the Heavy Ion Fusion Virtual National Laboratory.

DOI: $10.1103 /$ PhysRevSTAB.7.104201

PACS numbers: 29.27.Bd, 41.85.Ct, 41.85.Ew
\end{abstract}

\section{INTRODUCTION}

In the currently envisioned configurations for heavy ion fusion (HIF), it is necessary to longitudinally compress the beam bunches by a large factor after the acceleration phase and before the beam particles are focused onto the fusion target. In order to obtain enough fusion energy gain, the peak current for each beam is required to be of the order of $10^{3} \mathrm{~A}$, and the bunch length to be as short as $0.5 \mathrm{~m}$. However, to deliver the beam particles at the required energy, it is technically challenging to accelerate short bunches at high current. Because of the finite rise time of the accelerating waveform, it is more tractable to accelerate and transport beam bunches longer than $10 \mathrm{~m}$. In addition, short bunches have higher current (density) and therefore stronger space-charge effects which can increase the beam emittance and induce halo particle production.

The objective of drift compression [1-12] is to compress a long charge bunch after acceleration by imposing a negative longitudinal velocity tilt over the length of the beam in the beam frame. As a result, the bunch length is reduced as the beam drifts downstream, until the spacecharge force in the longitudinal direction becomes strong enough to remove the initial velocity tilt. Different longitudinal compression schemes have been studied. In Haber's study [2] and de Hoon et al.'s recent study [7,8], for example, the line density is uniform in the middle of the pulse but falls off at the ends, whereas in other recent studies [6,9-12], the line density is parabolic over the length of the pulse. Recently, a warm-fluid model has been developed to study the longitudinal dynamics of the drift compression $[9,10]$. It was shown that a self-similar solution with parabolic density profile can be used for drift compression, and a pulse shaping technique has also been demonstrated so that any initial pulse shape can be shaped into a parabolic one which can then be selfsimilarly compressed. Because the space-charge force increases as the beam is compressed, the beam size in the transverse direction will increase in a periodic quadrupole lattice. If an active control of the beam size is desired, a larger focusing force is needed to confine the beam in the transverse direction, and a nonperiodic quadrupole lattice along the beam path is necessary. In this paper, we describe the design of such a focusing lattice along with the final focusing magnets which will focus the beam onto the target. Because the beam parameters are evolving and the lattice is nonperiodic, the concept of a "matched" beam is not well defined. However, if the nonperiodicity is relatively weak, we can still find "adiabatically matched" solutions. Another important issue is that the drift compression and final focus lattice should work for the entire pulse, which may have different perveance and emittance for different slices. In particular, different slices should be focused onto the same focal spot at the target. This is very difficult with a fixed lattice. One solution is to use a time- 
dependent lattice which provides different focusing strength for different slices of the beam pulse [13]. We demonstrate that the entire pulse can be drift compressed and focused onto the same spot on the target by using four time-varying quadrupole magnets at the very beginning of the drift compression.

Two sets of beam parameters will be used in the present study, while the general methods are applicable to other parameter regimes and application areas. The first set of beam parameters is for a typical heavy ion fusion driver, where we consider a $\mathrm{Cs}^{+}$beam with rest mass $m=132.9$ a.u., kinetic energy $(\gamma-1) m c^{2}=$ $2.43 \mathrm{GeV}$, and initial beam half length $z_{b 0}=5.85 \mathrm{~m}$. Our goal is to compress the beam by a factor of 21.8 , i.e., $z_{b f}=z_{b 0} / 21.8=0.268 \mathrm{~m}$. The initial peak current is $I_{0}=103.4 \mathrm{~A}$. The second set of beam parameters is for the integrated beam experiment (IBX) currently being designed by the Heavy Ion Fusion Virtual National Laboratory. In the IBX, a $K^{+}$beam with rest mass $m=$ 39.1 a.u. is accelerated to $6 \mathrm{MeV}$ kinetic energy. The initial beam half length is $z_{b 0}=0.68 \mathrm{~m}$ before the drift compression, and the initial peak current is $I_{0}=0.563 \mathrm{~A}$. The final beam half length is $z_{b f}=0.068 \mathrm{~m}$ after the drift compression.

This paper is organized as follows. In Sec. II, we briefly review the self-similar parabolic longitudinal drift compression scheme developed using a set of onedimensional warm-fluid equations $[9,10]$. In Sec. III, a nonperiodic lattice for drift compression is designed along with the final focus magnets. Four time-dependent magnets at the upstream of drift compression are used so that the entire pulse can be focused onto the same focal point. In both Secs. II and III, we will use the set of beam parameters for a typical heavy ion fusion driver to demonstrate the basic approach and methods. The same approach is then applied to the IBX drift compression design in Sec. IV.

\section{LONGITUDINAL DYNAMICS}

In Refs. [9,10], a one-dimensional warm-fluid model was developed to describe the longitudinal dynamics of drift compression. For the longitudinal electric field, the conventional $g$-factor model is used with $e E_{z}=$ $-\left(g e^{2} / \gamma^{2}\right) \partial \lambda / \partial z$ and $g=2 \ln \left(r_{w} / r_{b}\right)$. Here, $e$ is the charge, $\lambda(t, z)$ is the line density, $r_{w}$ is the wall radius, and $r_{b}$ is the beam radius. In the space-charge-dominated regime, the $g$-factor model adopted here is consistent with the result recently derived by Davidson and Startsev [14]. We also allow for an externally applied focusing force $F_{z}=-\kappa_{z} z$. In the beam frame, the warm-fluid equations for the line density $\lambda(t, z)$, longitudinal velocity $v_{z}(t, z)$, and longitudinal pressure $p_{z}(t, z)$ are given by $[9,10]$

$$
\frac{\partial \lambda}{\partial t}+\frac{\partial}{\partial z}\left(\lambda v_{z}\right)=0
$$

$$
\begin{gathered}
\frac{\partial v_{z}}{\partial t}+v_{z} \frac{\partial v_{z}}{\partial z}+\frac{e^{2} g}{m \gamma^{5}} \frac{\partial \lambda}{\partial z}+\frac{\kappa_{z} z}{m \gamma^{3}}+\frac{r_{b}^{2}}{m \gamma^{3} \lambda} \frac{\partial p_{z}}{\partial z}=0 \\
\frac{\partial p_{z}}{\partial t}+v_{z} \frac{\partial p_{z}}{\partial z}+3 p_{z} \frac{\partial v_{z}}{\partial z}=0
\end{gathered}
$$

We treat $g$ and $r_{b}$ as constants for present purposes. Among all the self-similar solutions [9] admitted by the nonlinear hyperbolic partial differential equation system (1)-(3), the parabolic self-similar solution is the most suitable for the purpose of drift compression. This solution has the form of

$$
\begin{aligned}
& \lambda(t, z)=\lambda_{b}(t)\left(1-\frac{z^{2}}{z_{b}^{2}(t)}\right), \quad v_{z}(t, z)=-v_{z b}(t) \frac{z}{z_{b}(t)}, \\
& p_{z}(t, z)=p_{z b}(t)\left(1-\frac{z^{2}}{z_{b}^{2}(t)}\right)^{2}, \quad \frac{d z_{b}(t)}{d t}=-v_{z b}(t) .
\end{aligned}
$$

Following the derivation in [9], we obtain the familiar longitudinal envelope equation

$$
\frac{d^{2} z_{b}}{d s^{2}}+\frac{\kappa_{z}}{m \gamma^{3} \beta^{2} c^{2}} z_{b}-K_{z} \frac{1}{z_{b}^{2}}-\varepsilon_{l}^{2} \frac{1}{z_{b}^{3}}=0,
$$

where $s=\beta c t$ is the normalized time variable, $K_{z} \equiv$ $3 N_{b} e^{2} g / 2 m \gamma^{5} \beta^{2} c^{2}$ is the effective longitudinal self-field perveance, and $\varepsilon_{l} \equiv\left(4 r_{b}^{2} W / m \gamma^{3} \beta^{2} c^{2} N_{b}\right)^{1 / 2}$ is the longitudinal emittance. Here, $W=z_{b}^{2} p_{z b}=$ const. In the drift compression scheme considered in this section for a typical heavy ion fusion driver, the longitudinal emittance is taken to be $\varepsilon_{l}=1.0 \times 10^{-5} \mathrm{~m}$, and $K_{z}=2.88 \times 10^{-5} \mathrm{~m}$, corresponding to an initial peak current $I_{0}=103.4 \mathrm{~A}$, final half bunch length $z_{b f}=0.268 \mathrm{~m}$, and $g=0.81$. Of course, it is desirable to minimize the initial velocity tilt and the beam path length for the drift compression. However, smaller velocity tilt implies longer path length, which is obvious from the first integral of Eq. (6). In practice, it is necessary to study the trade-off between the velocity tilt and the beam path length. A detailed study of this subject can be found in Ref. [9]. After carefully balancing these competing factors, we choose to impose an initial longitudinal focusing force for $s<$ $150 \mathrm{~m}$ so that the beam acquires a velocity tilt $z_{b}^{\prime}=$ -0.0143 at $s=150 \mathrm{~m}$. The axial beam half length $z_{b}(s)$, obtained numerically from the longitudinal envelope equation (6), is plotted together with the velocity tilt $z_{b}^{\prime}(s)$ in Fig. 1 . The total beam path length is $s_{f}=502 \mathrm{~m}$.

\section{TRANSVERSE DYNAMICS}

For each slice in a bunched beam, the transverse dynamics in a quadrupole lattice is described approximately by the transverse envelope equation: 

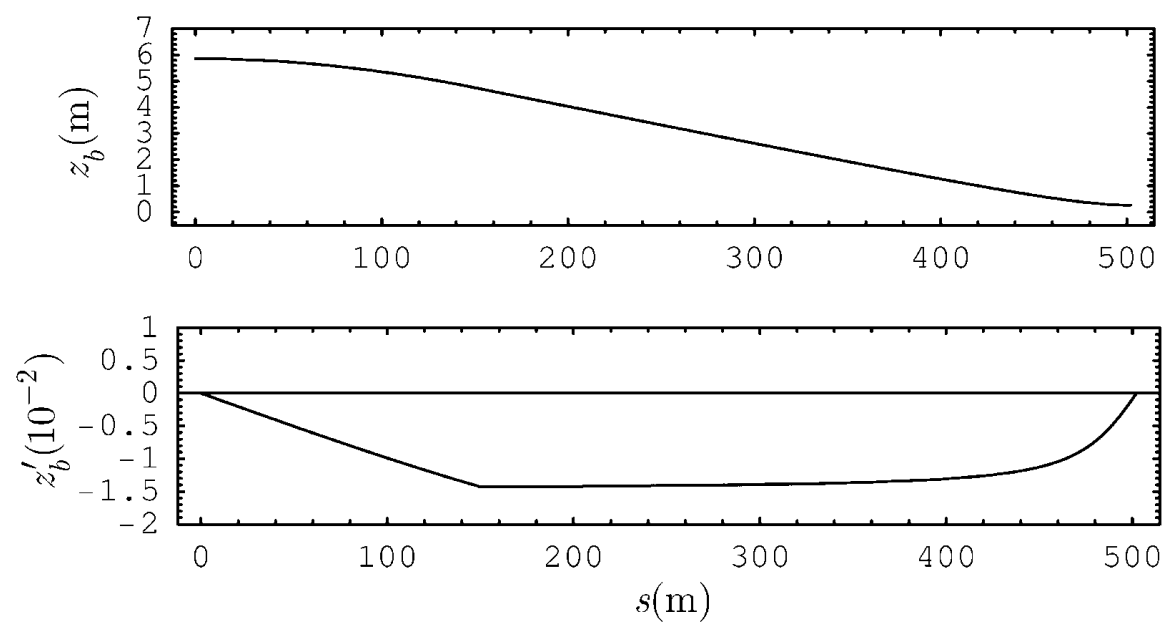

FIG. 1. Longitudinal drift compression of a heavy ion fusion beam.

$$
\begin{aligned}
& \frac{\partial^{2} a(s, z)}{\partial s^{2}}+\kappa_{q} a(s, z)-\frac{2 K(s, z)}{a(s, z)+b(s, z)}-\frac{\varepsilon_{x}^{2}}{a(s, z)^{3}}=0, \\
& \frac{\partial^{2} b(s, z)}{\partial s^{2}}-\kappa_{q} b(s, z)-\frac{2 K(s, z)}{a(s, z)+b(s, z)}-\frac{\varepsilon_{y}^{2}}{b(s, z)^{3}}=0,
\end{aligned}
$$

where $K(s, z) \equiv 2 e^{2} \lambda_{b}(s)\left[1-z^{2} / z_{b}^{2}(s)\right] / m \gamma^{3} \beta^{2} c^{2} z_{b}(s)$ is the effective perveance. Here $z$ is the longitudinal coordinate for different slices, and it enters the equations only parametrically. Because $K(s, z)$ is an increasing function of $s$, it is necessary to increase the strength of the quadrupole lattice coefficient $\kappa_{q}(s)$ along the beam path to actively control the expansion of the beam radius. Since the beam parameters are evolving and the quadrupole lattice is not periodic, the concept of a matched beam is not well defined. However, if the nonperiodicity is small, that is, if the quadrupole lattice changes slowly along the beam path, we can seek an adiabatically matched beam which, by definition, is locally matched everywhere.

We describe the design of a nonperiodic lattice which provides the required control of the beam radius when the beam is compressed, and equally important, minimizes the possibility of global mismatch. The designed drift compression and final focus lattice should apply for all of the slices in the bunched beam. In particular, each slice of the beam needs to be focused onto the same spot at the target. A fixed lattice designed for one slice of the beam (e.g., the central slice at $z=0$ ) will not focus other slices onto the same spot. Actually, most of the other slices cannot be focused at all due to their different perveance and emittance. We show that the goal can be achieved by designing a drift compression and final focus lattice for the central slice $(z=0)$, and then replacing four quadrupole magnets at the beginning of the drift compression by four time-dependent magnets whose strength varies around the design value for the central slice. The timedependent magnets essentially provide a slightly different focusing lattice for different slices.

First, we design the drift compression and final focus lattice for the central slice $z=0$. We assume that a lattice, which keeps both the vacuum phase advance and depressed phase advance constant, may have advantages with respect to the transverse dynamics. Lee [4] derived the expressions for the vacuum phase advance $\sigma_{v}$ and depressed phase advance $\sigma$ given by

$$
\begin{gathered}
2\left(1-\cos \sigma_{v}\right)=\left(1-\frac{2 \eta}{3}\right) \eta^{2}\left(\frac{B^{\prime}}{[B \rho]}\right)^{2} L^{4}, \\
\sigma^{2}=2\left(1-\cos \sigma_{v}\right)-K\left(\frac{2 L}{\langle a\rangle}\right)^{2} .
\end{gathered}
$$

Here, $\eta$ is the filling factor, $L$ is the half lattice period, $B^{\prime}$ is field gradient of the magnets, and $\langle a\rangle$ is the average beam radius. Assuming $\eta \ll 1$, we obtain

$$
\eta^{2}\left(\frac{B^{\prime}}{[B \rho]}\right)^{2} L^{4}=\text { const, } \quad K\left(\frac{2 L}{\langle a\rangle}\right)^{2}=\text { const, }
$$

for constant vacuum phase advance and constant depressed phase advance. For the drift compression scheme considered here for a typical heavy ion fusion driver, $K_{f} / K_{0}=21.8$. If we allow $\langle a\rangle$ to increase by a factor of 2.33, i.e., $\langle a\rangle_{f} /\langle a\rangle_{0}=2.33$, we obtain $L_{f} / L_{0}=1 / 2$, and $\left(\eta B^{\prime}\right)_{f} /\left(\eta B^{\prime}\right)_{0}=4$. We determine $K(s)$ from the solution of the longitudinal envelope equation. The value of $\langle a\rangle$ is determined from the solutions to Eqs. (7) and (8). For the lattice design, we need to specify $\eta, B^{\prime}$, and $L$. If we choose $L_{i}=L_{0} \exp \left[-(\ln 2) s_{i} / s_{f}\right]$, and $B_{i}^{\prime}=$ const, then from Eq. (11), $\eta_{i}=\eta_{0} \exp \left[(\ln 4) s_{i} / s_{f}\right]$, where $s_{i}=$ $\sum_{j=0}^{i-1} L_{j}$. We choose the exponential form for the nonperiodic lattice parameters to uniformly distribute the required variation of the lattice parameters according to their amplitudes. Other variation schemes may be effec- 
tive too, as long as they are smooth enough. We also choose self-consistently the following system parameters: vacuum phase advance $\sigma_{v}=72^{\circ}$, magnetic field gradient $B_{i}^{\prime}=31.70 \mathrm{~T} / \mathrm{m}$, initial half lattice period $L_{0}=$ $6.72 \mathrm{~m}$, and initial filling factor $\eta_{0}=0.0725$. The focusing strength of each magnet is $\hat{\kappa}=0.38 \mathrm{~m}^{-2}$. Let $N$ denote the total number of quadrupole magnet sets. From $s_{f}=\sum_{j=0}^{N-1} L_{j}$, we obtain $N=53$. The lattice design is illustrated in Fig. 2 together with the solutions to Eqs. (7) and (8). After determining the nonperiodic lattice layout, we search iteratively for the adiabatically matched solutions to Eqs. (7) and (8). The solutions plotted in Fig. 2 are adiabatically matched because the envelope is locally matched and contains no oscillations other than the local envelope oscillations. On the global scale, the beam radius increases monotonically. From the numerical solution shown in Fig. 2, the average beam size increases by a factor of 2 , which agrees with the design assumption.

The final focus magnets, consisting of four quadrupole magnets with different strength, will make the envelope converge in both directions at the exit from the final focusing magnet (i.e., both $a^{\prime}$ and $b^{\prime}$ are negative). Right after the final focusing magnet, the beam enters a neutralization chamber where the space-charge force is neutralized and the beam is focused onto a focal point at

$$
z_{\mathrm{fol}}=-\left.\frac{a}{\partial a / \partial s}\right|_{s=s_{\mathrm{ff}}}=-\left.\frac{b}{\partial b / \partial s}\right|_{s=s_{\mathrm{ff}}},
$$

where $z_{\text {fol }}$ is the distance between the focal point and the
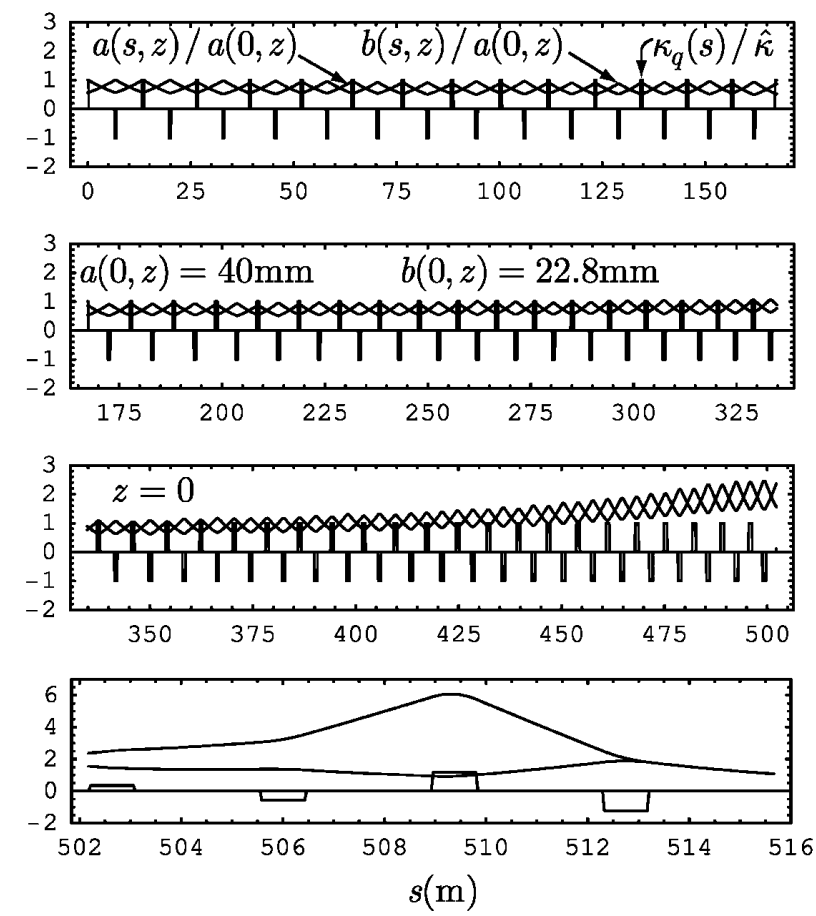

FIG. 2. Envelope dynamics for the central slice with $z=0$. exit of the last final focus magnet, and $s_{\mathrm{ff}}$ is the distance from the beginning of the drift compression to the exit of the last final focus magnet. It is necessary that $a /(\partial a / \partial s)$ and $b /(\partial b / \partial s)$ have the same value at $s=s_{\text {ff }}$ such that a focal point exists. The transverse spot size measured by the envelope amplitudes at the focal point $a_{\text {fol }}$ and $b_{\text {fol }}$ is determined by the emittance and incident angle at $s=s_{\mathrm{ff}}$,

$$
a_{\mathrm{fol}}=\left.\frac{\varepsilon_{x}}{\partial a / \partial s}\right|_{s=s_{\mathrm{ff}}}, \quad b_{\mathrm{fol}}=\left.\frac{\varepsilon_{y}}{\partial b / \partial s}\right|_{s=s_{\mathrm{ff}}} .
$$

For the central slice $z=0$, we obtain

$$
z_{\mathrm{fol}}=5 \mathrm{~m}, \quad a_{\mathrm{fol}}=b_{\mathrm{fol}}=1.2 \mathrm{~mm} .
$$

The required gradient strength for the four final focusing magnets is obtained by a four-dimensional rootsearching algorithm.

For other slices $(z \neq 0)$, our objective is to manipulate the beam and the magnets so that the beam pulse can be focused onto the same focal point with the same or smaller spot size,

$$
z_{\mathrm{fol}}=5 \mathrm{~m}, \quad a_{\mathrm{fol}} \approx b_{\mathrm{fol}} \lesssim 1.2 \mathrm{~mm} .
$$

To begin with, we notice that, in a fixed lattice, the following scaling holds

$$
K \rightarrow \alpha^{2} K, \quad a \rightarrow \alpha a, \quad b \rightarrow \alpha b,
$$

if the emittance is negligible or scales as $\varepsilon \rightarrow \alpha^{2} \varepsilon$. For the line density profile

$$
\lambda(s, z)=\lambda_{b}(s)\left[1-z^{2} / z_{b}^{2}(s)\right],
$$

the solution to the transverse envelope equations for all of the slices can be scaled down from that of the central slice according to

$$
\left(\begin{array}{c}
a(s, z) \\
b(s, z) \\
\partial a(s, z) / \partial s \\
\partial b(s, z) / \partial s
\end{array}\right)=\sqrt{1-z^{2} / z_{b}^{2}(s)}\left(\begin{array}{c}
a(s, 0) \\
b(s, 0) \\
\partial a(s, 0) / \partial s \\
\partial b(s, 0) / \partial s
\end{array}\right),
$$

provided the emittance is negligibly small or scales with the perveance according to $\left(\varepsilon_{x}, \varepsilon_{y}\right) \propto 1-z^{2} / z_{b}^{2}(s)$. However, the emittance in general is small but not negligible, and does not scale with the perveance. This implies that requirement (15) cannot be satisfied. In fact, during adiabatic drift compression or pulse shaping for an initially isothermal beam, the emittance scales with the beam size, i.e., $\varepsilon_{x} \propto a$ and $\varepsilon_{y} \propto b$. In this paper, we assume that the initial emittance scales with the beam size, and that for each longitudinal slice the normalized emittance is conserved during the drift compression and final focus. Therefore, the scaling in Eq. (17) and the requirement in Eq. (15) are not satisfied.

One solution to this difficulty is to vary the strength of four magnets in the very beginning of the drift compression for different $z$ such that the desired scaling holds at 
the end of the last magnet $\left(s=s_{\mathrm{ff}}\right)$,

$$
\left(\begin{array}{c}
a\left(s=s_{\mathrm{ff}}, z\right) \\
b\left(s=s_{\mathrm{ff}}, z\right) \\
\partial a\left(s=s_{\mathrm{ff}}, z\right) / \partial s \\
\partial b\left(s=s_{\mathrm{ff}}, z\right) / \partial s
\end{array}\right)=\sqrt{1-z^{2} / z_{b}^{2}\left(s=s_{\mathrm{ff}}\right)}\left(\begin{array}{c}
a\left(s=s_{\mathrm{ff}}, 0\right) \\
b\left(s=s_{\mathrm{ff}}, 0\right) \\
\partial a\left(s=s_{\mathrm{ff}}, 0\right) / \partial s \\
\partial b\left(s=s_{\mathrm{ff}}, 0\right) / \partial s
\end{array}\right) .
$$

Combined with Eqs. (12) and (13), Eq. (18) will guarantee satisfaction of the requirement in Eq. (15). It is a viable solution because the emittance and therefore the departure from the desired scaling is small. Numerically, the necessary variation of the strength of the magnets is found by a four-dimensional root-searching algorithm. Shown in Fig. 3 are the dynamics of $a(s, z)$ and $b(s, z)$ for $z / z_{b}(s)=0.96$, when the strength of the $3 \mathrm{rd}, 5 \mathrm{th}, 7 \mathrm{th}$, and 9 th magnets is modified to satisfy Eq. (18). The initial conditions are taken to be those in Eq. (17). As evident for the figure, small perturbations in the strength of the magnets introduce a small envelope mismatch in such a way that Eq. (18) is satisfied at $s=s_{\mathrm{ff}}$. We note that a similar scaling does not exist for $0<s<s_{\mathrm{ff}}$. Plotted in Fig. 4 is the gradient strength of the $3 \mathrm{rd}, 5 \mathrm{th}, 7 \mathrm{th}$, and 9 th magnets as functions of $z$ which are able to focus the entire beam onto the same focal point with the same spot size. As mentioned before, for the case shown in Figs. 3 and 4 we have assumed $\varepsilon_{x}=\varepsilon_{y} \propto \sqrt{1-z^{2} / z_{b}^{2}(s)}$, which is not a necessary condition for this method to work. In principle, we can use this method to correct any
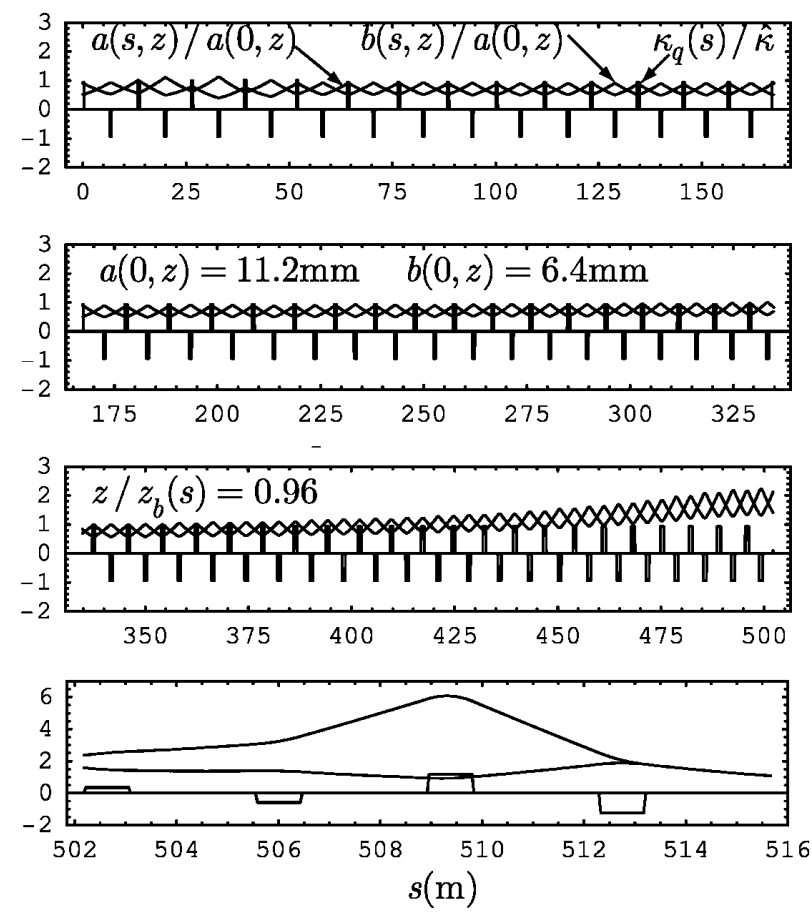

FIG. 3. Envelope dynamics for the slice near the front end of the beam pulse with $z / z_{b}(s)=0.96$. deviation from Eq. (18) due to any possible mechanism, such as momentum spread and magnet imperfections.

\section{DRIFT COMPRESSION FOR THE IBX}

The objective of the integrated beam experiment proposed by the Heavy Ion Fusion Virtual National Laboratory [15] is to investigate heavy ion fusion beam physics in an integrated manner using a scaled-down system. In particular, it is proposed to use IBX to study the drift compression physics, which is a necessary component in a heavy ion fusion driver. The ion beam used in IBX is a $K^{+}$beam with $6 \mathrm{MeV}$ kinetic energy. The initial beam half length is $z_{b 0}=0.68 \mathrm{~m}$, and the initial peak current is $I_{0}=0.563 \mathrm{~A}$. The final beam half length is $z_{b f}=0.068 \mathrm{~m}$ after drift compression.

For the longitudinal dynamics, after balancing the needs of reducing the beam path length and minimizing the initial velocity tilt, we have chosen the initial velocity tilt to be $z_{b 0}^{\prime}=-0.0621$. The numerical solution of the longitudinal envelope equation (6) is plotted in Fig. 5. In this design, the beam path length is $s_{f}=11.68 \mathrm{~m}$. The initial velocity tilt chosen here is considerably larger than that for the heavy ion fusion driver discussed in Sec. II. To minimize the cost of the IBX, it is required to design a drift compression scheme with beam path length in the range of $10 \mathrm{~m}$ or so.

For the transverse lattice, we choose to use a constant FODO lattice to reduce the cost and complexity. This is a reasonable design because the longitudinal compression ratio is 10, and according to Eq. (11), the beam size will increase by a modest factor of 3.16, i.e., $\langle a\rangle_{f} /\langle a\rangle_{0}=3.16$.

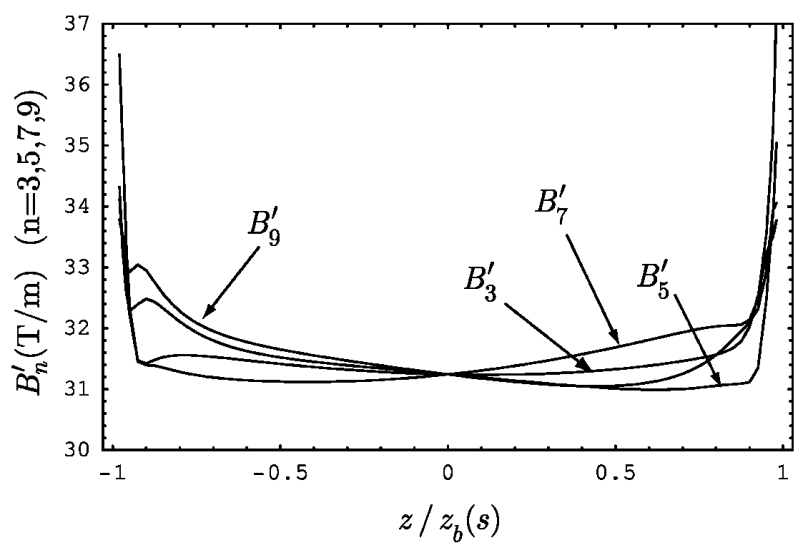

FIG. 4. Strength of the $3 \mathrm{rd}, 5$ th, 7th, and 9th magnets as functions of $z$. 

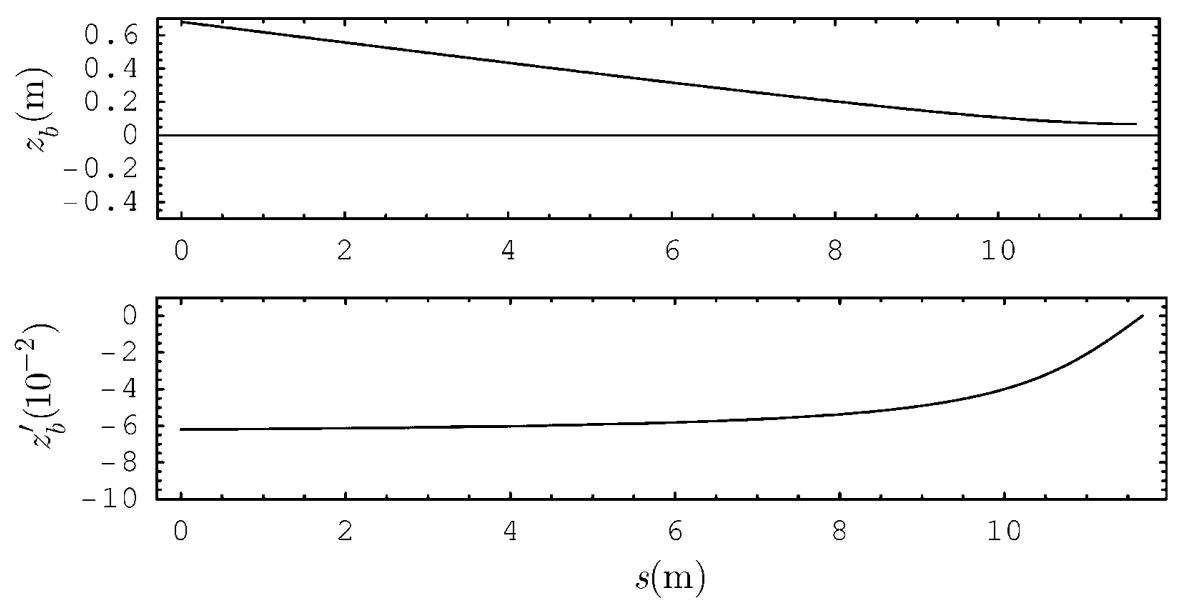

FIG. 5. Longitudinal drift compression of an IBX beam pulse.

Other system parameters are vacuum phase advance $\sigma_{v}=30^{\circ}$, magnetic field gradient $B_{i}^{\prime}=20 \mathrm{~T} / \mathrm{m}$, half lattice period $L=0.39 \mathrm{~m}$, and filling factor $\eta=0.375$. The numerical solutions of the transverse envelope equations are plotted in Fig. 6 for the central slice $(z=0)$, from which it is obvious that the average beam size increases by a factor close to 3.16, agreeing with the design assumption. We note that the long-bunch approximation is valid almost everywhere except at the very end of the drift compression, where the longitudinal size is comparable to the transverse size, and the $g$-factor model may need correction in this small region. Also plotted in Fig. 6 is the beam envelope during the final focus. Here, we have designed a two-magnet final focusing system to
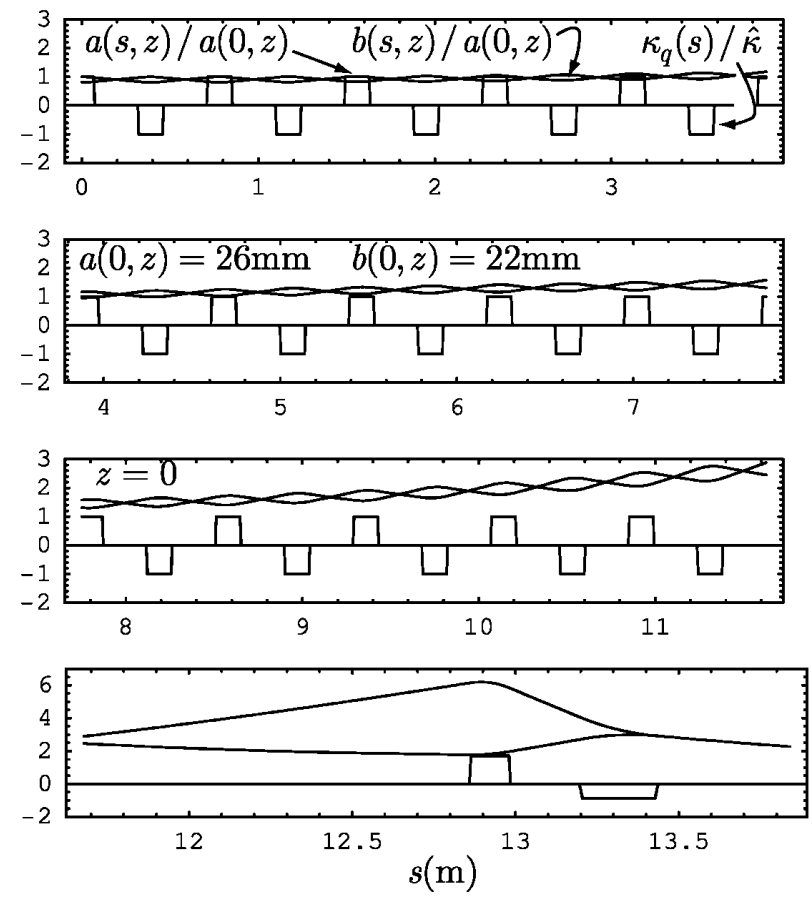

FIG. 6. Envelope dynamics for the central slice with $z=0$. minimize the cost. The final focal point and focal size are determined from Eqs. (12) and (13). For the central slice, these parameters are

$$
z_{\mathrm{fol}}=1.61 \mathrm{~m}, \quad a_{\mathrm{fol}}=b_{\mathrm{fol}}=2.95 \mathrm{~mm} .
$$

For other slices $(z \neq 0)$, we apply the same method discussed in Sec. III to design a lattice with four timevarying magnets in the upstream of the drift compression to focus the entire beam pulse onto the same focal point with the same or smaller spot size. As an example, the numerical solutions for the beam envelopes are shown in Fig. 7 for a slice near the beam front end with $z / z_{b}(s)=$ 0.96 , where the gradient strength of the $3 \mathrm{rd}, 4 \mathrm{th}, 5 \mathrm{th}$, and
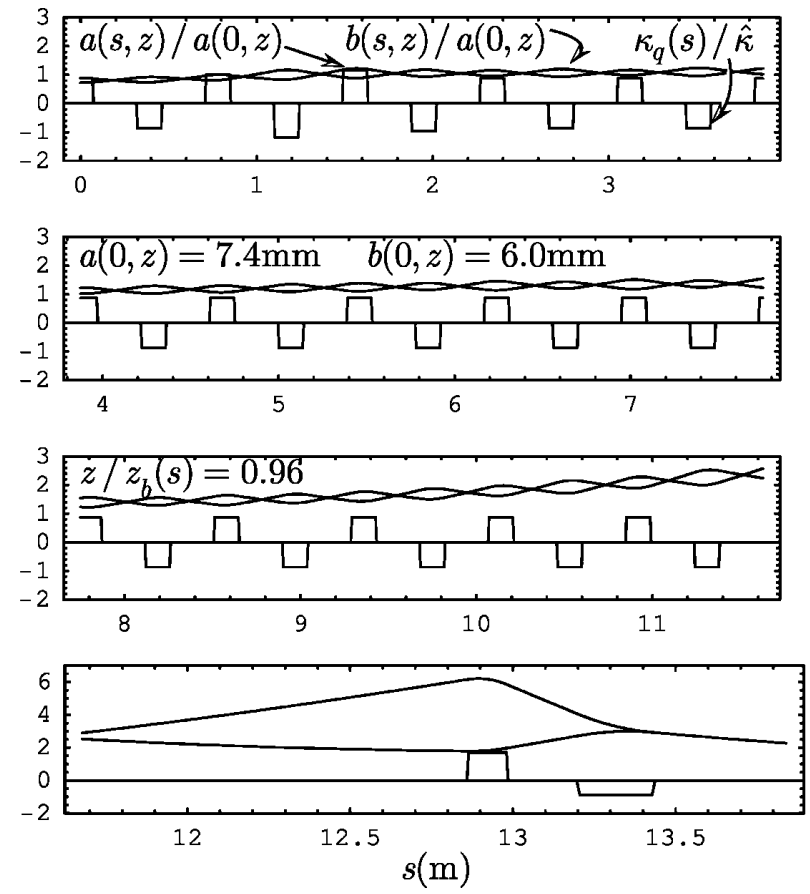

FIG. 7. Envelope dynamics for the slice near the front end of the beam pulse with $z / z_{b}(s)=0.96$. 


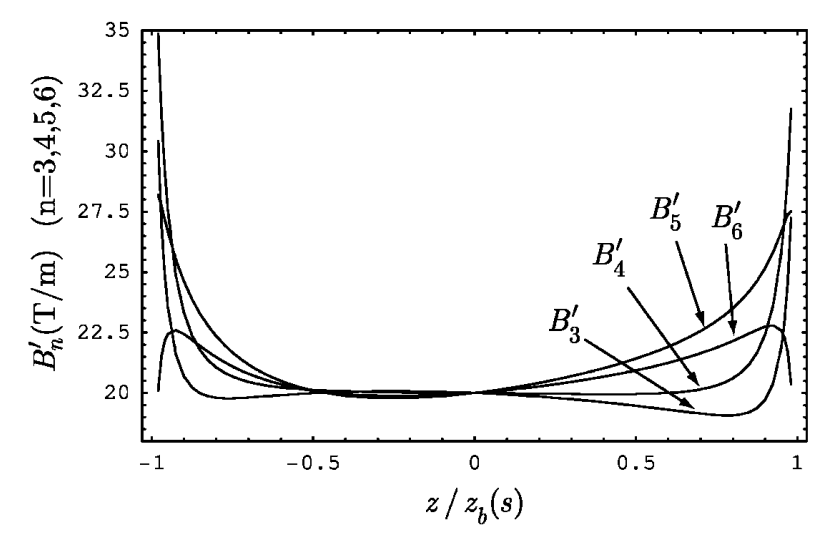

FIG. 8. Strength of the 3 rd, 4th, 5th, and 6 th magnets as functions of $z$.

6th magnets is modified in such a way that Eq. (18) is satisfied. Plotted in Fig. 8 is the gradient strength of the $3 \mathrm{rd}, 4 \mathrm{th}, 5 \mathrm{th}$, and 6 th magnets as functions of $z / z_{b}(s)$ which are able to focus the entire beam onto the same focal point with the same spot size.

\section{CONCLUSION AND FUTURE WORK}

We have studied the transverse dynamics of heavy ion beams during the drift compression and final focus phases. To accommodate the significant increase of space charge during the drift compression, a nonperiodic lattice is designed so that it is possible to actively control the transverse size of the beam. Different slices of the beam may have different perveance and emittance, which makes it impossible to design a fixed drift compression and final focus lattice for the entire beam. Four timedependent magnets are introduced in the upstream of the drift compression to focus the entire beam pulse onto the same focal spot. The nonperiodic, time-dependent lattice design in the present paper, combined with the longitudinal drift compression scheme developed in Refs. $[9,10]$, provide the elements of a leading-order drift compression scheme. The next-step investigation will be focused on second-order effects, such as emittance growth during drift compression, and the coupling between longitudinal and transverse dynamics. Large-scale particle simulation studies will help us to identify important higher-order effects and develop modifications to the leading-order design. New results in these areas will be reported in the future.

\section{ACKNOWLEDGMENTS}

This research was supported by the U.S. Department of Energy under the auspices of the Heavy Ion Fusion Virtual National Laboratory. We thank Dr. Alex Friedman, Dr. Simon Yu, and Dr. Grant Logan for constructive discussion and comments.

[1] D. Neuffer, Part. Accel. 11, 23 (1980).

[2] I. Haber, in Proceedings of the Symposium on Accelerator Aspects of Heavy Ion Fusion, Darmstadt, 1982 (GSI, Darmstadt, 1982), p. 372.

[3] I. Hofmann and I. Bozsik, in Proceedings of the Symposium on Accelerator Aspects of Heavy Ion Fusion, Darmstadt, 1982 (Ref. [2]), p. 362.

[4] E. P. Lee, Lawrence Berkeley Laboratory Report No. 19560, 1985.

[5] J. Bisognano, E. P. Lee, and J.W.-K Mark, Laser Program Annual Report No. 84, LLNL, 3-28, 1985.

[6] D. D.-M. Ho, S. T. Brandon, and E. P. Lee, Part. Accel. 35, 15 (1991).

[7] M. J. L. de Hoon, Ph.D. thesis, University of California at Berkeley, 2001.

[8] M. J. L. de Hoon, E. P. Lee, and J. J. Barnard, in Proceedings of 2001 Particle Accelerator Conference, Chicago, IL (IEEE, Piscataway, NJ, 2001), p. 729.

[9] H. Qin and R. C. Davidson, Phys. Rev. ST Accel. Beams 5, 034401 (2002).

[10] H. Qin and R. C. Davidson, Laser Part. Beams 20, 565 (2002).

[11] E. P. Lee and J. J. Barnard, Laser Part. Beams 20, 581 (2002).

[12] H. Qin, R. C. Davidson, J. J. Barnard, and E. P. Lee, in Proceedings of 2003 Particle Accelerator Conference, Portland, OR (IEEE, Piscataway, NJ, 2003), p. 2658.

[13] According to Dr. R. O. Bangerter, this method was first proposed by Dr. A.W. Maschke in an early International Symposium on Heavy Ion Inertial Fusion.

[14] R. C. Davidson and E. A. Startsev, Phys. Rev. ST Accel. Beams 7, 024401 (2004).

[15] J. J. Barnard et al., Laser Part. Beams 21, 553 (2003). 